\title{
Perfil fisiológico de canoístas do sexo feminino de alto nível competitivo
}

\author{
Fábio Yuzo Nakamura ${ }^{1,2}$ \\ Thiago Oliveira Borges 1,2 \\ Fabrício Azevedo Voltarelli ${ }^{3}$ \\ Luis Alberto Gobbo1,2 \\ Alvaro Acco Koslowiski1,4 \\ Maria Alice Rostom Mello ${ }^{3}$
}

https://doi.org/10.5628/rpcd.06.03.329

\begin{abstract}
RESUMO
O objetivo deste estudo foi caracterizar o perfil fisiológico de atletas de canoagem do sexo feminino de alto nível competitivo. Para isso, as atletas passaram por protocolos de testes para determinação: (1) da velocidade crítica (VCrit) e capacidade anaeróbia da canoagem ( $\left.\mathrm{CA}_{\text {Canoagem }}\right) ;(2)$ do limiar de lactato mínimo (LACmin) e; (3) do pico de lactato após esforços máximos de 130 e $500 \mathrm{~m}$. Os resultados mostraram que os valores de VCrit $(3,01-3,04 \mathrm{~m} / \mathrm{s})$ foram significantemente maiores que os de LACmin $(2,75 \pm 0,04 \mathrm{~m} / \mathrm{s})$. Já a $\mathrm{CA}_{\text {Canoagem variou entre }}$ 94-105 m. Não foram encontradas diferenças entre os valores de pico de lactato no sangue após os esforços de 130 m (10,5 $\pm 1,2 \mathrm{mM})$ e $500 \mathrm{~m}(10,2 \pm 0,8 \mathrm{mM})$. A conclusão foi que as atletas estudadas apresentaram altos níveis específicos de capacidade aeróbia e lática, quando comparadas com amostras de outros estudos na literatura.
\end{abstract}

Palavras-chave: canoagem, capacidade aeróbia, capacidade e potência lática.

\author{
${ }^{1}$ Grupo de Estudo das A daptações Fisiológicas ao \\ Treinamento (GEA FIT), Centro de Educação Física e \\ D esportos, Universidade Estadual de Londrina, \\ Londrina/PR \\ 2 Grupo de Estudo e Pesquisa em M etabolismo, Nutrição \\ e Exercício, Centro de Educação Física e D esportos, \\ U niversidade Estadual de Londrina, Londrina/PR \\ 3 Instituto de Biociências, Departamento de Educação \\ Física, U niversidade Estadual Paulista, Rio Claro/SP \\ ${ }^{4} \mathrm{C}$ entro $\mathrm{N}$ acional de Treinamento Canoagem Velocidade, \\ Confederação Brasileira de $\mathrm{C}$ anoagem
}

\section{ABSTRACT}

Physiological profile of high competitive level female kayakists

The aim of this study was to characterize the physiological profile of kayaking female athletes with high competitive level. For this, the athletes underwent testing protocols to determination of: (1) critical velocity (VCrit) and anaerobic kayaking capacity $\left(\mathrm{CA}_{\text {canoagem }}\right)$; (2) lactate minimum threshold (LAC min) and; (3) peak blood lactate after maximal efforts comprising the 130 and $500 \mathrm{~m}$ distances. The results showed that the $V C$ rit values $(3,01-3,04 \mathrm{~m} / \mathrm{s})$ were significantly greater than the $L A C$ min values $(2,75 \pm 0,04 \mathrm{~m} / \mathrm{s})$. The $C A_{\text {canoagem }}$ ranged between 94-105 $\mathrm{m}$. There were not found differences in the peak blood lactate after the efforts in $130 \mathrm{~m}(10,5 \pm 1,2 \mathrm{mM})$ compared to the $500 \mathrm{~m}(10,2 \pm 0,8 \mathrm{mM})$. The conclusion was that the athletes here investigated presented high levels of specific aerobic and lactic capacity, when compared to samples of other studies in the literature.

Key-words: kayaking, aerobic capacity, lactic capacity and power. 


\section{INTRODUÇÃO}

A canoagem é uma modalidade esportiva cujas distâncias competitivas (200, 500 e 1.000 m) exigem altas taxas de ressíntese de ATP para a realização de trabalho externo e interno, predominando as ações do segmento superior do corpo. Essa energia provém tanto do metabolismo aeróbio quanto do anaeróbio (15). Ressalta-se, no entanto, que as mulheres atualmente competem apenas na distância de $500 \mathrm{~m}$. Nesse sentido, estudos (6) têm apontado correlações significativas entre indicadores de capacidade e potência aeróbia (limiares metabólicos, consumo máximo de oxigênio - $\mathrm{VO}_{2} \max$ ) e anaeróbia (máximo défice acumulado de oxigênio - MAOD, teste de Wingate adaptado) em caiaque-ergômetro, e o desempenho nas regatas em linha. Alguns desses indicadores têm sido capazes de discriminar atletas de diferentes níveis competitivos (22) e gêneros (2). Outras investigações mostraram também que al guns atributos antropométricos e a força muscular $(6,11)$ são componentes importantes para a performance de canoístas.

Destaca-se que, com poucas exceções (21), a maior parte das avaliações funcionais feitas em canoístas foram conduzidas em caiaque-ergômetro. Assim, a transferência dos resultados para a água fica comprometida, sobretudo quando a intenção é utilizar os indicadores para a prescrição das cargas de treinamento. Além disso, a literatura carece de valores de referência para esses indicadores fisiológicos de performance em atletas de alto nível competitivo, sobretudo em mulheres.

O modelo de velocidade crítica $(13,14)$ foi adaptado com sucesso na canoagem, na medida em que o parâmetro velocidade crítica (VCrit) correlaciona-se positivamente com o desempenho de endurance de $6.000 \mathrm{~m}$ (17). Em adição, a VCrit apresenta sensibilidade aos efeitos específicos do treinamento em canoístas (dados não publicados). Outro modelo de determinação da capacidade aeróbia, o qual consiste na aplicação do protocolo de limiar de lactato mínimo (20), apesar de aparentemente coincidir com a VCrit, pois também se inclui dentre as abordagens indiretas para a detecção do máximo estado estável de lactato (MSSL), não foi ainda testado na canoagem.

Além disso, por falta de uma medida amplamente aceita em testes de campo, al guns autores têm ado- tado o pico de concentração de lactato sangüíneo como indicador de capacidade lática $(10,18)$. Esse índice também não foi testado na canoagem.

Portanto, o objetivo deste estudo foi caracterizar o perfil fisiológico de atletas femininas da seleção nacional de canoagem, padronizados em outras formas de exercício, como limiar de lactato mínimo e pico de lactato acumulado no sangue, além do modelo de velocidade crítica, ao desempenho em caiaque individual $\mathrm{K}-1$.

\section{METODOLOGIA}

\section{Amostra}

Participaram deste estudo oito canoístas do sexo feminino (idade: 18,0 \pm 2,3 anos; estatura: 160,0 \pm $6,1 \mathrm{~cm}$; massa corporal: $56,8 \pm 4,9 \mathrm{~kg}$ ), pertencentes à Seleção Brasileira de Canoagem, sediada no município de Caxias do Sul - RS. Todas as participantes, ou seus responsáveis, assinaram termo de consentimento livre e esclarecido para participar deste estudo. Elas estavam em período de preparação geral para a temporada competitiva de 2005. Todas as atletas passaram por uma bateria de testes no próprio caiaque, a saber: (1) testes de 500, 1.000 e 2.000 m para estimativa dos parâmetros do modelo de velocidade crítica; (2) testes de 130 e 500 m para estimativas do pico de concentração de lactato pós-esforço e; (3) teste com velocidades progressivas pós-indução de aumento da lactatemia sangüínea para estimativa da velocidade associada ao limiar de lactato mínimo.

Testes para estimativa dos parâmetros do modelo de velocidade crítica

Foram realizados testes em três distâncias para estimativa dos parâmetros do modelo de velocidade crítica (500, 1.000 e 2.000 m). Previamente aos testes máximos, as atletas realizaram aquecimento em ritmo livremente escolhido em distância $\sim 1.000 \mathrm{~m}$. Os testes foram realizados em dias diferentes, e procurou-se conduzi-los no mesmo período do dia e com as características climáticas semel hantes (preferencial mente sem vento forte a favor ou contra a trajetória das embarcações). Era solicitado que, ao sinal do avaliador, a atleta realizasse um tiro à máxima velocidade para a respectiva distância. As atletas foram avaliadas em grupos para motivá-las a tentar o melhor desempenho. $\mathrm{O}$ tempo foi registrado com 
um cronômetro manual com precisão de décimos de segundos.

As equações utilizadas para estimativa dos parâmetros foram: (1) função não linear velocidade-tempo; (2) função linear distância-tempo e; (3) função linear velocidade-1/tempo. As equações estão descritas abaixo, de acordo com Hill (9):

$$
\begin{aligned}
& \text { Tempo }=\mathrm{CA}_{\text {Canoagem }} /(\text { velocidade }- \text { VCrit }) \\
& \text { Distância }=\mathrm{CA}_{\text {Canoagem }}+\mathrm{VCrit} * \text { tempo } \\
& \text { Velocidade }=\text { VCrit }+\mathrm{CA}_{\text {Canoagem }} / \text { tempo }
\end{aligned}
$$

Onde $\mathrm{CA}_{\text {Canoagem }}$ é a capacidade anaeróbia da canoagem, medida em metros, e VCrit indica a vel ocidade crítica, em metros por segundo.

Testes para estimativa da capacidade lática (130 e 500 m) Foram realizados testes nas distâncias de 130 e 500 $\mathrm{m}$, em dias separados, para estimativa da capacidade lática. O teste de $500 \mathrm{~m}$ foi o mesmo realizado quando da estimativa dos parâmetros do modelo de velocidade crítica. A padronização do aquecimento foi semelhante ao utilizado no item anterior.

Novamente, solicitou-se que as canoístas desenvolvessem a maior velocidade possível para cada uma das distâncias.

Amostras de sangue ( $25 \mu \mathrm{l})$ foram retiradas do lóbulo da orel ha para anál ise da concentração de lactato em repouso, e a um, três, cinco e sete minutos após o término dos testes de 130 e $500 \mathrm{~m}$. Essas amostras foram coletadas a partir de uma pequena incisão feita com lancetas descartáveis, sendo transferidas para tubos capilares heparinizados. Logo após as coletas, o sangue era depositado em tubos eppendorf contendo $400 \mu$ de ácido tricloroacético (TCA) 4\%. O material era mantido em caixa de isopor com gelo para análise posterior.

No laboratório, a concentração de lactato sanguíneo foi determinada a partir de método enzimático (LDH $=$ lactato desidrogenase) proposto por Engles e Jones (4). Houve centrifugação das amostras a 2000 rpm durante dois minutos, e pipetagem de $100 \mu \mathrm{l}$ a partir do sobrenadante, em tubos de ensaio. Adicionou-se a este $500 \mu \mathrm{l}$ de reativo contendo NAD e LDH. As amostras foram agitadas e incubadas (banho-maria) durante 20 minutos à temperatura de 37 으. A leitura de lactato foi efetuada em aparelho espectrofotômetro no comprimento de onda de 340 $\mathrm{nm}$, com as conversões necessárias para obtenção da medida em $\mathrm{mM}$.

Foi obtido para cada atleta o pico de concentração de lactato após esforço máximo nas distâncias de 130 e $500 \mathrm{~m}$ (18). Essas medidas foram consideradas aproximações da capacidade lática.

Teste para estimativa do limiar de lactato mínimo (LACmin)

Após a indução de elevada lactatemia no teste de $500 \mathrm{~m}$, e pausa passiva de sete minutos, foi realizado um teste com velocidades progressivas para estimativa do LACmin. Os tempos médios para cada um dos estágios foram de $280 \pm 0 \mathrm{~s}, 267 \pm 7 \mathrm{~s}, 248 \pm 4$ s e $229 \pm 1 \mathrm{~s}$, para um percurso fixo de $700 \mathrm{~m}$, demarcado por meio de um retângulo dentro do lago em que foram feitas as coletas. Apenas uma das atletas foi capaz de completar mais um percurso adicional com duração de $217 \mathrm{~s}$. Assumiu-se que a distância fixada era adequada na medida em que permitia durações de estágios suficientes para a estabilização do lactato sangüíneo. Elas se aproximam das utilizadas no trabal ho original de Tegtbur et al. (20). O controle das velocidades era feito por dois dos avaliadores, que conduziam um barco regulado por sistema GPS, ditando o ritmo de deslocamento.

Após cada um dos estágios, amostras de sangue foram coletadas e tratadas no laboratório, conforme já mencionado no item anterior. As concentrações de lactato foram ajustadas a uma função polinomial de segunda ordem, para que o ponto mínimo da curva fosse determinado (figura 1). A velocidade associada a esse ponto foi considerada como sendo o LACmin da canoísta. Foi registrada também a concentração de lactato observada nessa intensidade. 
Figura 1 - Comportamento da concentração sangüínea de lactato durante o protocolo de velocidades progressivas para determinação do limiar de lactato mínimo (LACmin) de uma atleta.

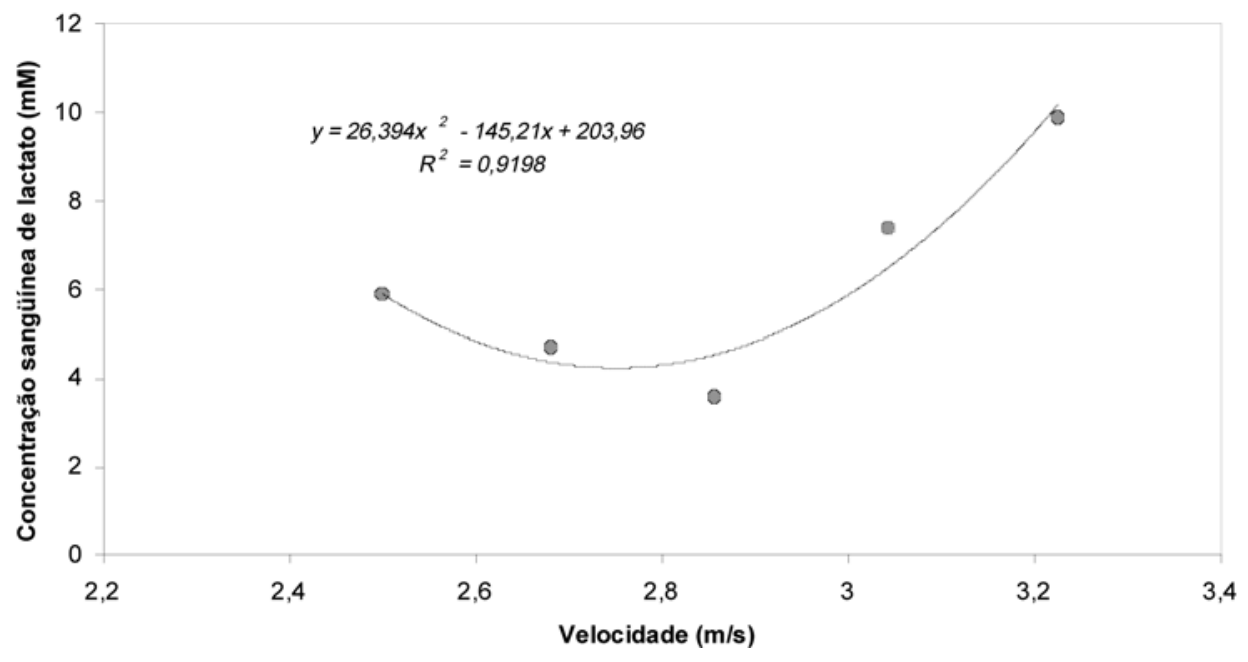

Análise estatística

Foi dado um tratamento descritivo a todos os dados referentes ao perfil fisiológico das canoístas (média \pm desvio padrão). Utilizou-se ANOVA para medidas repetidas para comparar as estimativas de VCrit por meio das três equações, em conjunto com LACmin. O mesmo procedimento foi utilizado para comparar as diferentes estimativas de $\mathrm{CA}_{\text {Canoagem. }} \mathrm{O}$ teste $\mathrm{t}$ para amostras dependentes foi empregado na comparação entre as concentrações de pico de lactato após os testes de 130 e 500 m. Em todos os casos, foi adotado um nível de significância de 5\%.

\section{RESULTADOS}

Em média, as canoístas deste estudo completaram os testes de 130, 500, 1.000 e $2.000 \mathrm{~m}$ em 31,9 \pm 1,8, $133,8 \pm 5,5,295,9 \pm 11,0$ e 629,8 \pm 26,6 s.

Na tabela 1 são apresentadas as estimativas de VCrit mediante aplicação das três equações preditivas, juntamente com a estimativa de LACmin. Houve diferença significativa entre $L A C m i n$ e as medidas de VCrit, sendo que entre as últimas não houve diferenças estatisticamente significativas. Em média, a concentração de lactato associada à ocorrência do LACmin foi de 3,78 $\pm 1,24 \mathrm{mM}$.
Tabela 1. Valores médios de velocidade crítica (VCrit) e da velocidade associada ao limiar de lactato mínimo (LACmin.) das canoístas pertencentes à amostra estudada $(n=8)$.

\begin{tabular}{|c|c|c|c|}
\hline \multicolumn{3}{|c|}{ VCrit [m/s] } & \\
\hline Equação 1 & Equação 2 & Equação 3 & LACmin [m/s] \\
\hline $3,01 \pm 0,14$ & $3,02 \pm 0,13$ & $3,04 \pm, 012$ & $2,75 \pm 0,04^{*}$ \\
\hline
\end{tabular}

* diferente das estimativas de VCrit $(p<0,05)$.

Na figura 2 está apresentada a comparação entre as estimativas de $\mathrm{CA}_{\text {Canoagem }}$ por meio das três equações previstas pelo modelo. Apesar da tendência à diferença ( $p=0,081)$, os valores foram próximos a 100 $\mathrm{m}$, em média.

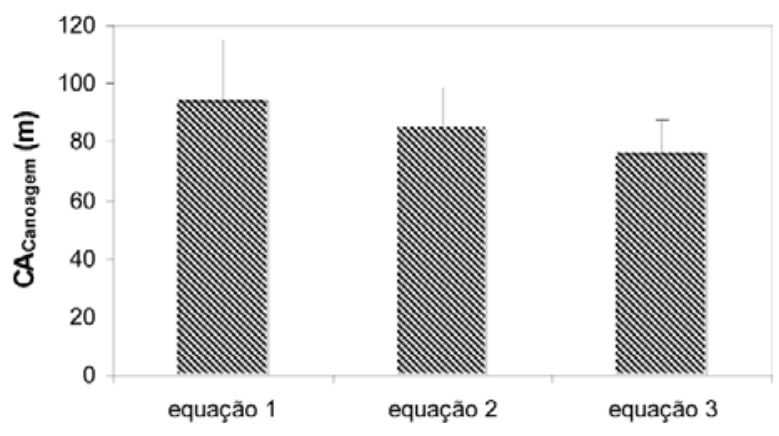

Figura 2. Valores médios da capacidade anaeróbia da canoagem $\left(\mathrm{CA}_{\text {canoagem }}\right)$ das canoístas pertencentes à amostra estudada. 
Não houve diferença no pico de lactato alcançado após os testes de 130 e $500 \mathrm{~m}$. No teste mais curto, a concentração de pico foi equival ente a 10,5 \pm 1,2 $\mathrm{mM}$, ao passo que no teste mais longo o valor atingido foi de 10,2 $\pm 0,8 \mathrm{mM}$.

\section{DISCUSSÃO}

O nível competitivo das atletas por nós investigadas era elevado. O tempo médio para $500 \mathrm{~m}$ foi de 133,8 $\pm 5,5 \mathrm{~s}$, fora do período competitivo. Esse tempo era inferior em termos de desempenho ao apresentado pela amostra estudada por Bishop et al. (1) (121,6 \pm 4,9 s), composta por canoístas do sexo feminino, representantes da equipe da Austrália nas categorias Junior e Sênior (média de 23,0 \pm 5,0 anos de idade). Cabe ressaltar que em nosso estudo as atletas eram mais jovens, contando com atletas de 14 anos de idade (18,0 $\pm 2,3$ anos, em média).

Em investigações anteriores, foram real izados testes em ergômetro de braços para anal isar o desempenho mecânico e fisiológico de canoístas (16) e nadadores (7). No estudo de Nakamura et al. (16), observou-se altas correlações entre a PCrit estimada no ergômetro de braços e a VCrit estimada nos caiaques, sendo a amostra composta por canoístas do sexo masculino de uma equipe local. Medidas de capacidades fisiológicas específicas na própria embarcação K-1 ainda são escassas.

As estimativas de VCrit apresentadas pelas atletas a partir das três equações matematicamente equivalentes não foram estatisticamente diferentes. $O$ mesmo ocorreu com as estimativas de $\mathrm{CA}_{\text {Canoagem. }}$. Estudos têm mostrado que a VCrit é uma aproximação válida do máximo estado estável de lactato em canoístas (5), sendo também correlacionada ao desempenho em distância de 6.000 m (17). Estudos em cicloergômetro mostram que a capacidade de trabal ho anaeróbio (CTAnaer) apresenta alta correlação com o máximo défice acumulado de oxigênio (3).

Dessa forma, a VCrit tem sido utilizada como parâmetro de capacidade aeróbia e a $\mathrm{CA}_{\text {Canoagem, }}$ de capacidade anaeróbia. Os valores encontrados nas atletas deste estudo (3,01-3,04 m/s) foram equivalentes aos reportados por $\mathrm{N}$ akamura et al. (16), em uma amostra de atletas do sexo masculino de uma equipe de nível nacional $(3,06-3,10 \mathrm{~m} / \mathrm{s})$. Portanto, considera-se que esses valores sejam relativamente altos. Já os valores de $\mathrm{CA}_{\text {Canoagem }}$ na presente amostra (94-105 $\mathrm{m}$ ) foram superiores à da equipe masculina (62-74 m).

Havia, no entanto, a expectativa de que as estimativas de VCrit correspondessem ao LACmin, uma vez que a descrição fisiológica do LACmin assemel ha-se ao da VCrit (13,21). Ao contrário do que se esperava, a velocidade de $\operatorname{LACmin}(2,75 \pm 0,04 \mathrm{~m} / \mathrm{s})$ foi menor que os valores de VCrit determinada por cada uma das equações, não se tendo encontrado correlações significativas.

Nossos resultados se al inham em parte aos de Simões et al. (19), os quais mostraram também que para um grupo de corredores o LACmin $(281,0 \pm$ $14,8 \mathrm{~m} / \mathrm{min})$ foi menor que a VCrit $(292,1 \pm 17,5$ $\mathrm{m} / \mathrm{min}$ ). No entanto, houve correlação significativa $(r=0,896)$ entre os índices. Havia estado estável de lactato e manutenção de corrida no LACmin por 30 min. Não foi testada a manutenção na VCrit, mas foi mostrado que essa intensidade foi a que melhor se associou à velocidade média mantida em prova de 10 $\mathrm{km}$. Cabe salientar, no entanto, que pelo fato do estudo de Simões et al. (19) ter sido conduzido tendo corredores como amostra, os resultados não podem ser diretamente comparados aos nossos. Todavia, observa-se um padrão de diferenças entre a VCrit e o LACmin.

Algumas limitações deste estudo devem ser mencionadas. Não realizamos medidas diretas do máximo estado estável de lactato, para comparação da VCrit e LACmin com uma medida amplamente aceita pela sua validade. Além disso, o número de pontos utilizados (quatro, em média) para determinação da curva para detecção do ponto mínimo da lactatemia foi relativamente reduzido em relação ao utilizado em trabal hos anteriores (12,20). Isso pode ter influenciado nos resultados. Tanto Tegtbur et al. (20) quanto Macl ntosh et al. (12) mostraram com número maior de pontos para a curva (seis a nove) que o LACmin corresponde ao máximo estado estável de lactato, além de apresentar correlação com a performance em provas de longa duração (12).

Não houve diferença na concentração de pico de lactato entre os testes de $130 \mathrm{~m}(10,5 \pm 1,2 \mathrm{mM})$ e 500 $\mathrm{m}(10,2 \pm 0,8 \mathrm{mM})$. Isso mostra que mesmo um teste de duração relativamente curta ( $31,9 \pm 1,8 \mathrm{~s}$ ), como é o caso do esforço máximo em $130 \mathrm{~m}$, pode gerar estimativas de pico de lactato as quais se apro- 
ximam sobremaneira dos maiores valores individuais alcançados em teste mais longo ( $133,8 \pm 5,5 \mathrm{~s}$ ). Os valores reportados neste estudo com relação aos valores de pico de lactato não foram significativamente diferentes daqueles reportados por atletas de alto nível após provas de corrida de $100 \mathrm{~m}(8,5 \pm$ $0,8 \mathrm{mM})$ e de $200 \mathrm{~m}(10,3 \pm 0,8 \mathrm{mM})$ (8). Portanto, o nível de lactatemia de pico pode ser considerado alto entre as canoístas, sobretudo pelas diferenças de massa muscular mobilizada em relação aos corredores. No entanto, como não há correlação entre a concentração de pico de lactato com a performance $(8,18)$, essa medida ainda precisa ser mais bem compreendida. Estudos longitudinais são necessários para determinar a sensibilidade dessas medidas derivadas da produção de ácido lático em resposta a diferentes formas de treinamento de canoagem.

Em síntese, neste estudo evidenciou-se a possibilidade de utilização de protocolos de testes aeróbios e anaeróbios padronizados em outras formas de exercício, adaptados à canoagem. A VCrit e o LACmin não foram semelhantes, e ainda permanece a questão sobre qual deles se aproxima mais do máximo estado estável de lactato. No entanto, particularmente a VCrit parece ser elevada nas atletas estudadas. Além disso, as atletas parecem tolerar elevados níveis de acidose em testes de capacidade lática, sobretudo ao se considerar a quantidade de massa muscular envolvida.

\section{CORRESPONDÊNCIA}

\section{Fábio Yuzo N akamura}

Grupo de Estudo das Adaptações Fisiológicas ao Treinamento

Centro de Educação Física e Desportos Universidade Estadual de Londrina

Rod. Celso Garcia Cid, km 380

Campus Universitário

CEP 86051-990 - Londrina, PR

Brasil

fabioy_nakamura@yahoo.com.br 


\section{REFERÊNCIAS}

1. Bishop D (2000). Physiological predictors of flat-water kayak performance in women. Eur J A ppl Physiol 82:91-97.

2. Bunc V, Heller J, Leso J, Sprynarová S, Zdanowicz R (1987). Ventilatory threshold in various groups of highly trained athletes. Int J Sports M ed 8: 275-280.

3. Chatagnon M, Pouilly J-P, Thomas V, Busso T (2005). Comparison between maximal power in the power-endurance relationship and maximal instantaneous power. Eur A ppl Physiol 94:711-7.

4. Engels RC, Jones JB (1978). Causes and elimination of erratic blanc in enzymatic metabolic assays involving the use of NAD in alkaline hydrazine buffers: improved conditions for assay of L-glutamate, L-lactate and other metabolites. A nal Biochem 88: 475-484.

5. Fontes EB, Nakamura FY, Gobbo LA, Altimari LR, Melo JC, Carvalho FO, Okano AH, Borges TO, Silva SG, Cyrino ES (2005). Does critical velocity represent maximal steady state lactate in canoe/ kayak flatwater? The FIEP Bulletin 75:427-30.

6. Fry RW, Morton AR (1991). Physiological and kinanthropometric attributes of elite flatwater kayakists. M ed Sci Sports Exerc 23:1297-1301.

7. Guglielmo LGA, Denadai BS (2001). Validade do ergômetro de braço para a determinação do limiar anaeróbio e da performance aeróbia de nadadores. Revista Portuguesa de Ciências do D esporto 1:7-13.

8. Hautier CA, Wouassi D, Arsac LM, Bitanga E, Thiriet P, Lacour JR. (1994). Relationship between postcompetittion blood lactate concentration and average running velocity over 100-m and 200-m races. Eur J A ppl Physiol 68: 508-513.

9. Hill DW (1993). The critical power concept: A review. Sports M ed 16: 237-254.

10. Jacobs I (1986). Blood lactate: Implications for training and sports performance. Sports M ed 3:10-25.

11. Liow DK, Hopkins WG (2003). Velocity specificity of weight training for kayak sprint performance. M ed Sci Sports Exerc 35:1232-1237.

12. Maclntosh BR, Esaú S, Svedahl K (2002). The lactate minimum test for cycling: Estimation of the maximal lactate steady state. Can J A ppl Physiol 27: 232-249.

13. Monod H, Scherrer J (1965). The work capacity of a synergic muscular group. Ergonomics 8:329-338.

14. Moritani T, Nagata A, DeVries HA, Muro M (1981). Critical power as a measure of physical work capacity and anaerobic threshold. Ergonomics 24:339-350.

15. Nakamura FY, Borges TO, Sales OR, Cyrino ES, Kokubun E (2004). Estimativa do custo energético e contribuição das diferentes vias metabólicas na canoagem de velocidade. Rev Bras M ed Esporte 10:70-77

16. Nakamura FY, Borges TO, Brunetto AF, Franchini $E$ (2005). Correlação entre os parâmetros do modelo de potência crítica no cicloergômetro de membros superiores e no caiaque. Rev Bras Ci M ov 13:41-48.

17. Nakamura FY, Borges TO, de-Oliveira FR, Caldeira LFS, Bertuzzi RCM, Matsushigue KA (2006). Predição do desempenho aeróbio na canoagem a partir da aplicação de diferentes modelos matemáticos de velocidade crítica. R ev Bras M ed Esporte 12: in press.

18. de-Oliveira FR, Lima-Silva AE, Nakamura FY, Kiss MAPDM, Gevaerd MS (2005). Testes de pista para avaliação da capacidade lática de corredores velocistas de alto nível. Rev Bras M ed Esporte 11: in press.

19. Simões HG, Denadai BS, Baldissera V, Hill DW, Campbell CSG (2005). Relationship and significance of lactate minimum, critical velocity, heart rate deflection and $3000 \mathrm{~m}$ track-tests for running. J Sports M ed Phys Fitness 45: in press.

20. Tegtbur U, Busse MW, Braumann KM (1993). Estimation of an individual equilibrium between lactate production and catabolism during exercise. M ed Sci Sports Exerc 25: 620-627.

21. van Someren KA, Phillips GR, Palmer GS (2000). Comparison of physiological responses to open water kaya king and kayak ergometry. Int J Sports M ed 21: 200-204.

22. van Someren KA, Palmer GS (2003). Prediction of 200-m sprint kayaking performance. Can J A ppl Physiol 28:505-517. 